\title{
Spinal cord injury patients with spasticity and pressure sores: Preliminary report on reconstruction with botulinum toxin treated muscle flaps
}

\author{
Luca Negosanti ${ }^{1}$, Giorgio Sanguinetti ${ }^{1}$, Luca Gaiani $^{2}$, Micaela Battilana ${ }^{1}$, Rita Capirossi ${ }^{1}$, Siriana Landi ${ }^{1}$ and Rossella Sgarzani $^{3 *}$ \\ ${ }^{1}$ Multi-specialized Care Unit, Montecatone Rehabilitation Institute, Imola (BO), Italy \\ ${ }^{2}$ Orthopedic Surgery, Imola Hospital, Imola (BO), Italy \\ ${ }^{3}$ Plastic Surgery, AUSL Romagna, Cesena, Italy
}

\begin{abstract}
Background: Pressure sores are frequent complications in spinal cord injured patients (SCI). Unfortunately, spasticity may be associated to SCI, muscle spasms can be very intense in the post-operative period after pressure sore treatment and may cause wound dehiscence.

Aim of the study is to investigate the efficacy of botulinum toxin (BTX) in preventing muscle flap spasms and complications in pressure sores treatment.

Materials and methods: Prospective, observational, single center study on consecutive adult spinal cord injury patients affected by spasticity and pressure sore, with indication to surgical debridement and reconstruction with muscle flap. Muscle flap was prepared by injection of 100 units of BTX-A 10 days preoperatively.

Collected data include patient and wound information, post-operative need for increased dose of anti-spasticity medications compared to the preoperative period, time to complete wound healing, follow up at 6 months.

Results: Five adult patients with SCI, spasticity and trochanteric pressure sore were included in the study. All the patients were male, median age was 48 . All the patients were paraplegic, and the etiology was spinal cord trauma. All sores were IV degree. Osteomyelitis was confirmed histologically in all the patients. None of the patients needed an increased dose of anti-spasticity medications, compared to the preoperative period. A complete healing of the wound was observed in all the cases 3 weeks after surgery. No recurrences were observed at a six months follow-up.
\end{abstract}

Conclusion: BTX-A can be a useful method to prevent complications in SCI patients and pressures sores, when a muscle flap is planned.

\section{Introduction}

Pressure sores are frequent complications in spinal cord injured patients (SCI) [1]. After wide soft and bony tissues debridement, the reconstruction can be achieved with several methods and muscle flaps are often used. Unfortunately, spasticity may be associated do SCI, due to the upper motor neuron syndrome [2]. More than $80 \%$ of SCI patients present spasticity [3], causing major additional disability: intense muscle spasms can be triggered by various stimula such as heat/ cold and bladder distention [4]. The symptoms are difficultly controlled by medications. In our experience muscle spasms can be very intense in the post-operative period after pressure sore treatment. When the reconstruction is performed with a muscle flap, spasms may cause early loosening of the stitches, flap dehiscence, prolonged healing time and possible failure of the reconstruction. Our first experience with Botulin Toxin (BTX) in muscle flaps, was a salvage procedure in a patient operated elsewhere for a sacral Marjolin's ulcer and reconstructed with bilateral gluteus myocutaneous flaps. In the postoperative period the patient presented an important spasticity with central dehiscence of the flaps. The patient was admitted at Montecatone Rehabilitation Institute and underwent peri-lesional intramuscular injection of 100 units of BTX-A in 4 points. A second reconstructive surgery was planned after BTX injection, but it was not necessary because we observed a relatively rapid reepithelization after 7 days from injection, after the onset of the paralyzing effect on the muscles, and probably the vasodilatation effect. The wound was completely healed after two weeks. Based on this experience, we designed the present study to investigate the efficacy of botulinum toxin in preventing muscle flap spasms and complications in pressure sores treatment.

\section{Materials and methods}

We present a prospective, observational, single center study on consecutive adult spinal cord injury patients affected by spasticity and pressure sore with indication to surgical debridement and reconstruction with muscle flap, treated at Montecatone Rehabilitation Institute between August 2018 and December 2018. According to our institutional surgical protocol, ischial sores and sacral sores are treated with wide bony and soft tissues debridement and immediate reconstruction with one local fasciocutaneous flap, while trochanteric sores are treated in two stages. The first surgical procedure consists in

${ }^{*}$ Correspondence to: Rossella Sgarzani, Chirurgia Plastica, Ospedale Bufalini, via Ghirotti 286, 47521 Cesena (FC), Italy, E-mail: rossellasgarzani@libero.it

Key words: botulin toxin type a, spinal cord lesion, pressure sore, spasticity, muscle flap

Received: June 18, 2019; Accepted: July 24, 2019; Published: July 31, 2019 
debridement and application of topical negative pressure therapy, while the second one is the reconstruction with muscle or musculocutaneous flaps (rectus femuralis, vastus lateralis or gracilis) in order to fill in the dead space. All the patients enrolled in the study presented therefore a trochanteric sore.

The muscle flap was prepared by injection of BTX-A: 100 units of BTX-A were injected into the muscle to be transferred in two different points of it. The injection was planned 10 days preoperatively in order to observe a complete effect during surgery. Collected data included age, sex, level and cause of SCI, grade of the pressure sore, histologic diagnosis of ostemyelitis, flap used for the reconstruction, post-operative need for increased dose of anti-spasticity medications compared to the preoperative period, time to complete wound healing with stitches removal and rehabilitation to the sitting position, follow up at 6 months.

\section{Results}

Five adult patients with SCI, spasticity and trochanteric pressure sore were included in the study. All the patients were male, median age was 48 (IQR 36-63). All the patients were paraplegic and the etiology was spinal cord trauma. All sores were extended to the bone (IV degree according to the European Pressure Ulcer Advisory Panel classification). All the patients underwent a two-stages surgery: the first procedure consisted in a wide debridement of the soft and bony tissues, with multiple biopsies for histology and culture, and it was followed by a 3 weeks period of topical negative pressure therapy. Osteomyelitis was confirmed histologically in all the patients. 100 units of BTX-A were injected into each muscle to be transferred in all the cases 10 days before the reconstructive time. In the second procedure the reconstruction was achieved with rectus femoralis muscle flap with skin graft in 3 cases, with a combined rectus femoralis muscle flap and vastus lateralis musculo-cutaneous flap in 1 case and with a combined rectus femoralis muscle flap, vastus lateralis musculo-cutaneous flap and gracilis muscle flap in 1 case. None of the patients needed an increased dose of antispasticity medications compared to the preoperative period. A complete healing of the wound was observed in all the cases 3 weeks after surgery. According to our institutional protocol, after 3 uneventful weeks, all the stitches were removed, and the patients started the rehabilitation to the sitting position. No recurrences were observed at a six months followup (medium follow up of 8 months, range 6 months- 10 months).

\section{Discussion}

Botulinum toxin type A injected intramuscularly acts on the neuromuscular junction in striated muscles, to inhibit the release of acetylcholine [5]. Its applications on muscolar function are well known in comestic medicine, treatment of spasticity, strabismus, benign essential blepharospasm [6]. The effects on the muscle has been applied also to animation deformities after mammary reconstruction with submuscolar implants [7] (Figure 1).

Moreover BTX-A blocks the cholinergic receptors at the glandular level and has proved its efficacy in the treatment of epiphora, hypersalivation, hyperhidrosis $[6,8]$. Multiple studies on animal models have suggested that BTX-A also has a vasodilation effect probably by autonomic nerve block. It is hypothesized that BTX-A acts by blocking norepinephrine and other vasoconstriction substances in the neuromuscular junction in the arterial smooth muscle, thus promoting vasodilation and consequently increasing blood supply in cutaneous flaps [9-11].
BTX-A protects against primary ischaemia of skin flaps, moreover it helps to salvage poor reperfusion in secondary ischaemia of skin flaps [12]. BTX-A may play a protective role in presence of comorbidies such as diabetes or smoke exposure [13]. Segreto et al. recently published a review the literature on the use of botulinum toxin A and B in flap surgery animal models, the toxin seems to play a potential role in improving flap survival. Perivascular injections targeting each vascular pedicle are preferred in cases of free flaps or axial flaps; subdermal injections are favorable in cases of random pattern skin flaps; and injections should be performed 7 days before flap elevation [14].

In our study the predominant effect of BTX-A is on the muscolar reversible denervation of intrafusal and extrafusal muscle fibers. The clinical effect of the toxin appears 24 to 72 hours following administration and lasts for 2 to 6 months according to the dosage (Figure 2).

The use of BTX-A infiltrations is a valid, not surgical, low cost and low risk procedure to treat muscle spasms. It is an easy procedure with a temporary effect but safely repeatable and reproducible; it avoids further surgical procedures and shows tolerable latency with satisfactory outcomes [15]. This procedure was effectively reported in head and neck reconstruction [16]. In 1997 it was used in eyelid reconstruction

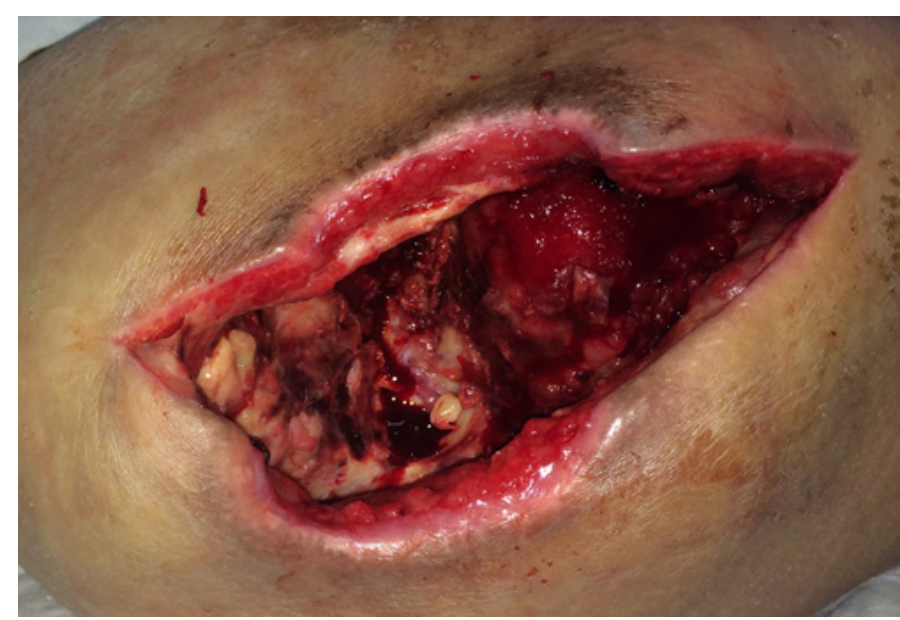

Figure 1. Preoperative picture of a male patient, 43 years old, paraplegic, affected by a wide trocanteric undermined pressure sore

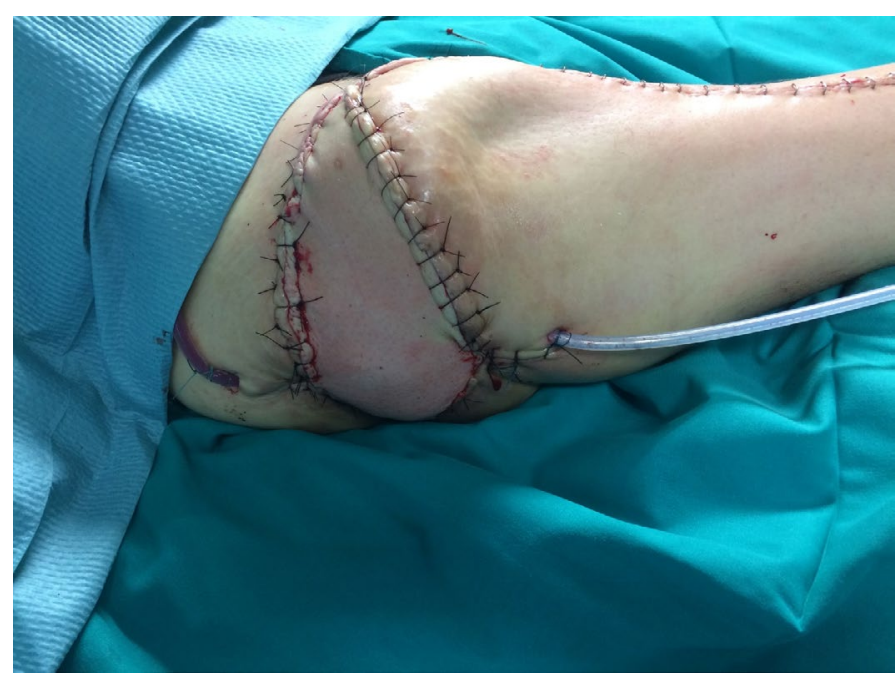

Figure 2. Post-operative picture after a reconstruction with botulinum toxin treated rectus femoralis muscle flap and vastus lateralis musculo-cutaneous flap 
to promote wound immobilization and prevent wound dehiscence in patients at risk of wound-healing complications [17].

In the pedicled muscle flaps, BTX-A can be used to prevent the detachment of the muscle flap $[5,18]$; it was also reported in expanded myocutaneous flaps [19]. The application in muscle flaps surgery in SCI patients was not reported in the literature. Denervation of the flap may be dangerous without magnification. It requires an extension of the pedicle dissection that might be dangerous if the reconstructive surgeon is not a trained microsurgeon, with possible lesion of the vascular pedicle. Moreover, denervation causes long term muscle atrophy with reduction of the protective thickness of the transferred tissues ${ }^{[5]}$. Botulinum toxin is a temporary alternative that can be useful to stabilize muscle flap avoiding denervation and long-term muscle atrophy. In our small series of patients, we observed good results. Further case-control studies are needed to compare complications in treated and not treated patients.

\section{Conclusion}

Spasticity is a frequent complication in SCI patients and it can affect the results of muscle flap reconstruction for pressure sores. BTX-A can be a useful method to prevent complications, or eventually to treat post-operative complications. Further case-control studies are needed to confirm our results.

\section{References}

1. Scheel Sailer A, Wyss A, Boldt C, Post MW, Lay V (2013) Prevalence, location, grade of pressure ulcers and association with specific patient characteristics in adult spinal cord injury patients during the hospital stay: a prospective cohort study. Spinal Cord 51: 828-833.

2. Lance J (1980) Pathophysiology of spasticity and clinical experience with baclofen. In: Lance J, Feldman R, Young R, Koella, W (ed's) Spasticity: Disordered Motor Control. Yearbook; Chicago, pp: 185-204.

3. Levi R, Hultling C, Seiger A (1995) The stockholm spinal cord injury study: 2. Associations between clinical patient characteristics and post-acute medical problems. Paraplegia 33: 585-594.

4. Little J, Micklesen P, Umlauf R, Britell C (1989) Lower extremity manifestations of spasticity in chronic spinal cord injury. Am J Phys Med Rehabil 68: 32-36.
5. Celik E, Tercan M, Uzunismail A, SaÄŸlam A (2006) Versatility of botulinum toxin: a use in stabilization of pedicled muscle flaps. Plast Reconstr Surg 117: 462-467. [Crossref]

6. Alsuhaibani AH, Eid SA (2018) Botulinum toxin injection and tear production. Curr Opin Ophthalmol 29: 428-433. [Crossref]

7. Figus A, Mazzocchi M, Dessy LA, Curinga G, Scuderi N (2009) Treatment of muscular contraction deformities with botulinum toxin type A after latissimus dorsi flap and subpectoral implant breast reconstruction. J Plast Reconstr Aesthet Surg 62: 869-875. [Crossref]

8. Lovato A, Restivo DA, Ottaviano G, Marioni G, Marchese-Ragona R (2017) Botulinum toxin therapy: functional silencing of salivary disorders. Acta Otorhinolaryngol Ital 37: 168-171. [Crossref]

9. Berry MG, Stanek JJ (2012) Botulinum neurotoxin A: a review. J Plast Reconstr Aesthet Surg 65: 1283-1291. [Crossref]

10. Neumeister MW, Chambers CB, Herron MS, Webb K, Wietfeldt J, et al. (2009) Botox therapy for ischemic digits. Plast Reconstr Surg 124: 191-201. [Crossref]

11. Morris JD, Jobling P, Gibbins IL (2002) Botulinum neurotoxin A attenuates releases of norepinephrine but not NPY from vasoconstrictor neurons. Am J Physiol Heart Circ Physiol 283: H2627-H35.

12. Huang L (2018) Beneficial effect of botulinum toxin A on secondary ischaemic injury of skin flaps in rats. Br J Oral Maxillofac Surg 56: 144-147. [Crossref]

13. Camargo CP, Jacomo AL, Battlehner CN, Lemos M, Saldiva PH, et al. (2015) Botulinum toxin type A on cutaneous flap viability in diabetic and tobacco-exposed rats. Acta Cir Bras 30: 639-645. [Crossref]

14. Segreto F, Marangi GF, Signoretti M, Cazzato V, Giorgino R, et al. (2019) The Use of Botulinum Toxin in Flap Surgery: A Review of the Literature. Surg Innov [Crossref]

15. Burgen AS, Dickens F, Zatman LJ (1949) The action of botulinum toxin on the neuromuscular junction. J Physiol 109: 10-24. [Crossref]

16. Rosales R, Arimura K, Takenaga S, Osame M (1996) Extrafusal and intrafusal muscle effects in experimental botulinum toxin-A injection. Muscle Nerve 19: 488-496.

17. Choi JC, Lucarelli MJ, Shore JW (1997) Use of botulinum A toxin in patients at risk of wound complications following eyelid reconstruction. Ophthalmic Plast Reconstr Surg 13: 259-264.

18. Trignano E, Dessy LA, Fallico N, Rampazzo A, Bassiri B, et al. (2012) Treatment of pectoralis major flap myospasms with botulinum toxin type A in head and neck reconstruction. J Plast Reconstr Aesthet Surg 65: 23-28

19. Chenwang D, Shiwei B, Dashan Y, Qiang L, Bin C, et al. (2009) Application of botulinum toxin type A in myocutaneous flap expansion. Plast Reconstr Surg 124: 1450-1457. [Crossref]

Copyright: (C2019 Negosanti L. This is an open-access article distributed under the terms of the Creative Commons Attribution License, which permits unrestricted use, distribution, and reproduction in any medium, provided the original author and source are credited. 